\title{
Metal-insulator transition and the character of the hole impurity bands in ferromagnetic GaMnAs disordered dilute magnetic semiconductor
}

\author{
R. da Silva Neves ${ }^{1}$, A. Ferreira da Silva ${ }^{1}$ and R. Kishore ${ }^{2}$ \\ ${ }^{1}$ Instituto de Física, Universidade Federal da Bahia, \\ 40210340 Salvador, Bahia, Brazil \\ ${ }^{2}$ Instituto Nacional de Pesquisas Espaciais -INPE/LAS \\ 12210970 São José dos Campos, São Paulo, Brazil.
}

\begin{abstract}
The study of ferromagnetic transition of $\mathrm{Ga}_{1-\mathrm{x}} \mathrm{Mn}_{\mathrm{x}} \mathrm{As}$ dilute magnetic semiconductor (DMS) is much of interest mainly due to the potential application in spintronic devices. Based on the mean field approach we present the average contribution of the hole spins by considering the holes in an impurity band (IB) and the critical concentration for the metal-insulator transition (MIT) in this semiconductor. In order to calculate the mean configuration of spins of impurities $\mathrm{Mn}^{+2}$ we use a formalism proposed for a spatial disordered system. The results for the metallic densities around the MIT transition are compared to experimental results and other theoretical findings.
\end{abstract}

\section{INTRODUCTION}

The discovery of the ferromagnetic transition at temperature $T_{C}$ of $\mathrm{Ga}_{1-\mathrm{x}} \mathrm{Mn}_{\mathrm{x}} \mathrm{As}$ exceeding $100 \mathrm{~K}$, is much of interest to investigate the physical properties of dilute magnetic semiconductor (DMS) mainly in view of the potential application in spintronic devices[1]. In our study, we consider that the ions $\mathrm{Mn}^{+2}$ impurities replacing the Ga sites in GaAs semiconductor [2] have a null local moment angular, momentum for spin $\mathrm{S}=$ $5 / 2$ and holes moderately bounded. Based on the mean field approach we calculate the average contribution of the hole spins by considering the holes in an impurity band (IB) and the critical concentration for the metal-insulator transition (MIT) in this semiconductor. In order to calculate the mean configuration of spins of impurities $\mathrm{Mn}^{+2}$ we used a formalism proposed for spatial disorder[3]. The best results, showed for intermediate and high concentrations (metallic phase systems), agree well with experimental results and other theoretical results[4,5], indicating a behavior character ferromagnetic phases. We may consider that our method is very useful in understanding the physical properties this magnetic material.

\section{METAL-INSULATOR TRANSITION}

In Mott's original model, the metal-insulator (MIT) transition occurs at a value of impurity critical concentration $\mathrm{N}_{\mathrm{c}}$, given by the relationship[6]

$$
N_{c}^{1 / 3} a^{*} \approx 0.25
$$

where $a^{*}$ is the effective Bohr radius of the system. 
In the Mott-Hubbard picture the MIT trasition is given by[7-9]

$$
\frac{\Delta \omega}{U}=1.15
$$

where $\Delta \omega$ is the unperturbed impurity bandwidth in units of $E_{b}$ and $U$ is the intra impurity coulomb interaction energy or Hubbard $U$, given by $U=0.96 E_{b}$ ( $E_{b}$ being the binding energy of a single acceptor system) $[10,11] . \Delta \omega$ is related to the hopping integral energy $T$, with adjacent sites $i$ and $j$, as $[9,10]$

$$
\Delta \omega=2|\langle T\rangle|
$$

where $\langle T(R)\rangle$, defined as the avarege hopping energy, is given by $[9,10]$

$$
\langle T(R)\rangle=\int T(R) P(R) d R
$$

In the equation above $T(R)$ and $U$ are given by [12]

$$
T(R)=\int \psi_{i}(\vec{r}) H_{1} \psi_{j}\left(\vec{r}-\vec{R}_{j}\right) d \vec{r}
$$

and

$$
U=\int\left|\psi\left(\vec{r}_{1}\right)\right|^{2}\left|\psi\left(\vec{r}_{2}\right)\right|^{2} \frac{e^{2}}{\varepsilon\left|\vec{r}_{1}-\vec{r}_{2}\right|} d \vec{r}_{1} d \vec{r}_{2}
$$

where $H_{1}$ is the one-particle Hamiltonian in the effective mass theory including the kinetic energy operator and the coulomb interaction of the negatively charged acceptor ion and the hole, and $\psi\left(\vec{r}-\vec{R}_{j}\right)$ is the simple hydrogenic wave function for the acceptor ground state at the randomly located site $\vec{R}_{j}$ and $\varepsilon$ is the dielectric constant of the host material. It is worth to write as well Eq.(5) as

$$
t(R)=t_{i j}=t\left(\left|R_{i}-R_{j}\right|\right)=2\left(1+R / a^{*}\right) \exp \left(-R / a^{*}\right) R y^{*} .
$$

In Eq.(4) we have used the random like Poisson distribution $P(R)$ of acceptor with the probability that the nearest acceptor neighbor lies at a distance $R$, in units of the effective Bohr radius $a^{*} . P(R)$ is written

$$
P(R)=\frac{3 R^{2}}{R_{a}^{3}}\left[1+\left(\frac{R}{R_{a}}\right)^{3}\right]^{-1},
$$

where 


$$
R_{a}=\left(\frac{4 \pi N_{a}}{3}\right)^{-1 / 3}
$$

and $N_{a}$ is the acceptor impurity concentration.

The value of $a^{*}$ is calculated from the experimental binding energy $E_{b}$ through the relation

$$
a^{*}=\frac{7.2}{\varepsilon E_{b}}
$$

where $a^{*}$ is given in Angstroms, $E_{b}$ in $\mathrm{eV}$ and $\varepsilon$ is the relative dielectric constant of the host material. Eqs. 3 to 9 show that the values of $U$ and $H_{1}$ are both related to binding energy $E_{b}$ of the acceptor impurity. Using the above equation we calculate the values of $a^{*}$ and $N_{c}$. Using $a^{*}=7.8 A$ and $\mathrm{E}_{\mathrm{b}}=112.4 \mathrm{meV}$ we found the following results, $N_{c}$ (Mott) $=3.3 \times 10^{19} \mathrm{~cm}^{-3}$ and $\mathrm{N}_{\mathrm{c}}$ (Mott-Hubbard) $=2.7 \times 10^{19} \mathrm{~cm}^{-3}$. These values correspond to about $1 \%$ of $\mathrm{N}_{\mathrm{Mn}}=1-5 \times 10^{20} \mathrm{~cm}^{-3}$ [13] and can be compared to $\sim 10^{18}$ $\mathrm{cm}^{-3}$ for p-type GaAs:C obtained from both methods described above and by using a Generalized Drude Approach and experimental conductivity[14].

\section{DISORDERED CONFIGURATION}

The real DMS carriers mediating the ferromagnetic interaction between local moments are generally likely to be far from in the valence band of the host semiconductors material. They are, in all likelihood, extended or bound in the impurity (or the defect) band, which form in the presence of the Mn dopants. There is a great deal of direct experimental support for the relevance of this impurity band picture for DMS ferromagnetism, and first principle band structure calculations also confirm the impurity band nature of the carriers active in DMS ferromagnetism. Both the impurity nature of the carrier system and presence of strong charge and spin disorder in the system (due to the random Mn dopants as well as other defects, disorder, and impurity invariably present in DMS materials) make carriers localization a relevant issue in DMS ferromagnetism.

According to this picture the model Hamiltonian for DMS in the Tight Binding picture is written as

$$
H=\sum_{i \sigma} t_{i} c_{i \sigma}^{\dagger} c_{j \sigma}+\sum_{i j \sigma} t_{j} c_{i \sigma}^{\dagger} c_{j \sigma}+\sum_{i j} J_{i j} S_{i} \cdot s_{j}+F(H)
$$

where $c_{i \sigma}^{\dagger}$ and $c_{i \sigma}$ are the creation and annihilation operators for holes and $J_{i j}$ is the antiferromagnetic interaction between spins of holes and Mn ions. We shall consider this model given by Eq.(1) as posed by Berciu and Bhatt[14] but here for $F(H)=0$. Now treating the third term in mean field approximation as

$$
S_{i} \cdot s_{j}=S_{M n} s_{j}^{z}+s_{h} S_{i}^{z}-S_{M n} s_{h}
$$


where $S_{M n}=\left\langle S_{i}^{z}\right\rangle$ and $s_{h}=\left\langle s_{j}^{z}\right\rangle$ are to configurationally averaged values of $\mathrm{Mn}$ and hole spin and thus independent of the site index.

We may obtain $s_{h}$ by considering the Green's function. For this specific case

$$
G_{i j \sigma}(t)=-i \theta<\left[c_{i \sigma}(t), c_{i \sigma}^{\dagger}(t)\right]_{+}>
$$

By considering the Fourier transform

$$
G_{i j \sigma}(t)=(1 / 2 \pi) \int_{-\infty}^{\infty} G_{i j \sigma}(\omega) \exp (-i \omega t) d t
$$

we get equation of motion of the Green's function $G_{i j}(\omega)$ as

$$
\left(\omega-\varepsilon_{i \sigma}\right) G_{i j \sigma}(\omega)=\delta_{i j}+\sum_{l} t_{i l} G_{l j \sigma}(\omega)
$$

where $\varepsilon_{i \sigma}=t_{i}+(\sigma / 2) S_{M n} J_{\text {eff }}$. We shall assume that $t_{i}=0$ (this assumption consistent with Berciu and Bhatt, they assumed $u(i)=0$ in their paper). From Eq.(10), we can write

$$
z G_{m n \sigma}(\omega)=\delta_{m n}+\frac{1}{z} t_{m n}+\sum_{v+1} \frac{1}{z^{v+1}} \sum_{l_{1} l_{2} \ldots l_{v}} t_{m l_{1}} t_{l_{1} l_{2}} \ldots t_{l_{v-1} l_{v}} t_{l_{v} n}
$$

where $z=\left(\omega-\varepsilon_{i \sigma}\right)$ and every $l_{i}$ extends for all impurity sites. Making the average over the ensemble of all settings in the equation (14)

$$
z\left\langle G_{m n \sigma}(\omega)\right\rangle=\delta_{m n}+\frac{1}{z} t_{m n}+\sum_{v=1}^{\infty} \sum_{\mu=1}^{v} \int \frac{N \mu}{z^{v+1}} t\left(\vec{R}_{m p_{1}}\right) \ldots t\left(\vec{R}_{p n}\right) d \vec{R}_{1} \ldots d \vec{R}_{\mu}
$$

where $\mu$ is the total number of intermediates sites for a given $\mu$ and $\eta$, we have all the sets possible of $\left(p_{1}, p_{2}, \ldots, p_{v}\right)$. Each site of impurity is denoted for a point which is associated with a factor of $\frac{1}{z}$ for each transfer from $n$ to $m$ site denoted for $t_{m n}$. Each term of the sum can be described by a possible path of the $i$ site, through $v$ impurities, so that only $\mu$ really distinct and returning to the $i$ site, as shown

$$
\begin{gathered}
\zeta_{\sigma}=\frac{1}{1-\eta_{\sigma}}=\infty=\infty+\beta_{0}+\beta_{+}+\cdots \\
\eta_{\sigma}=1-\frac{1}{\zeta_{\sigma}}=\frac{N \zeta^{ \pm}}{8 \pi^{2} z^{2}} \int \frac{t^{2}}{1-\frac{N \zeta_{\sigma} t(\vec{k})}{z}} d \vec{k}=\infty=\infty_{+}+\varnothing_{+}^{\infty}
\end{gathered}
$$


Now considering the off-diagonal disorder in the hopping integral $t_{i j}$ and following Matsubara and Toyozawa, we get the configurationally average diagonal Green's function from equations (14-16) $[3,15]$, where

$$
t(\vec{k})=\int t(\vec{R}) \exp (i \vec{k} \cdot \vec{R}) d \vec{R}
$$

is the Fourier transform of

$$
t_{i j}=t_{0}\left(1+\alpha\left|\vec{R}_{i}-\vec{R}_{j}\right|\right) \exp \left(-\alpha\left|\vec{R}_{i}-\vec{R}_{j}\right|\right)
$$

The density of states per impurity for spin $\sigma$ is

$$
D_{\sigma}(\omega)=-(1 / \pi) \lim _{\varepsilon \rightarrow 0} \operatorname{Im}<G_{i i \sigma}(\omega-i \varepsilon)>
$$

From the density of states we can obtain the $s_{h}$ as

$$
s_{h}=(1 / 2) \sum_{\sigma} \sigma \int d \omega D_{\sigma}(\omega)(\exp (\beta(\omega-\mu))+1)^{-1}
$$

where the Fermi enrgy $\mu$ can be obtained from the number of holes per impurity $p=n_{c} / n_{i}$ as

$$
p=\sum_{\sigma} \int_{-\infty}^{\mu} d \omega D_{\sigma}(\omega)
$$

As in Berciu and Bhatt [4] $p=c_{h} / c_{M n}=n_{c} / n_{i}, \quad n_{i}=c_{M n} ; \quad n_{c}=c_{h} \quad$ and $p=c_{h} / c_{M n}=n_{c} / n_{i}$.

\section{RESULTS AND DISCUSSIONS}

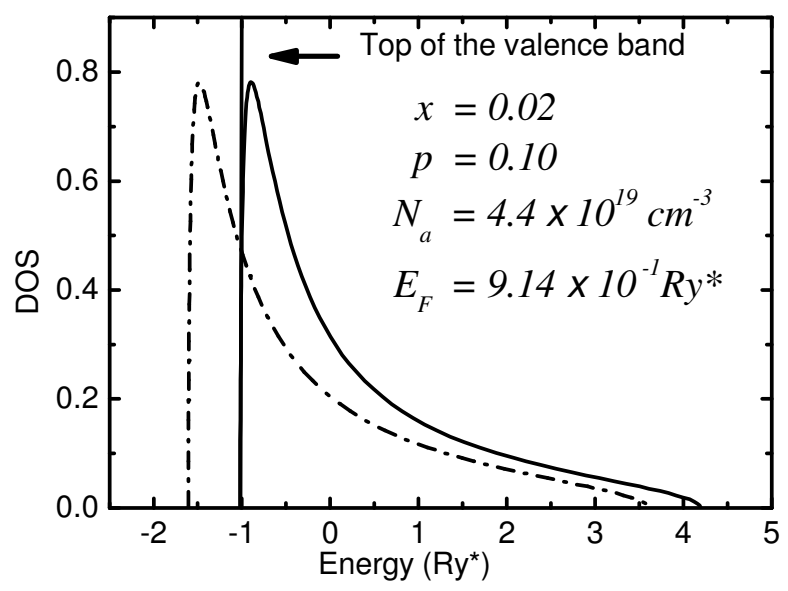

Figure 1. Impurity density of states (DOS) obtained for acceptor concentration $N_{a}$ around the MIT transition for $\mathrm{Ga}_{1-\mathrm{x}} \mathrm{Mn}_{\mathrm{x}} \mathrm{As}$. $\mathrm{Ry}^{*}=112 \mathrm{meV}$. 


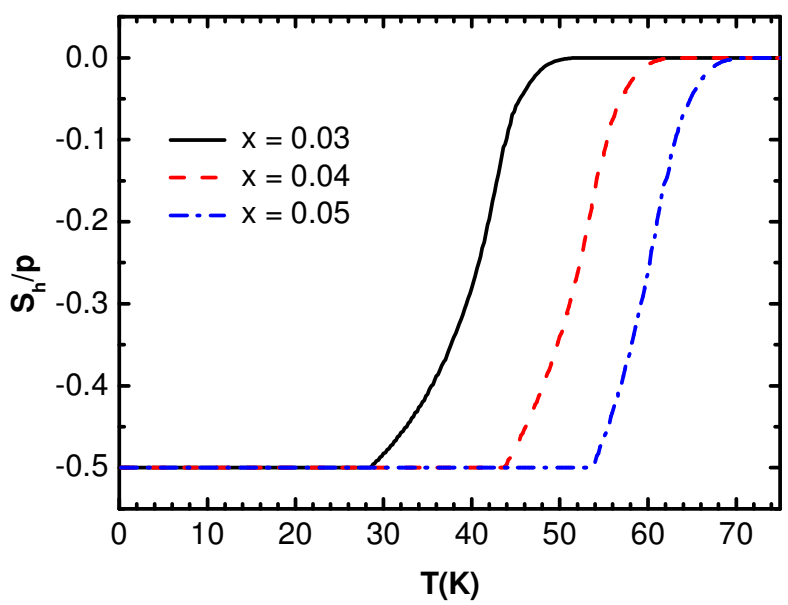

Figure 2. The average spin $s_{h}$ spin per charge carrier for $N_{M n}$ concentration $x=3-5 \%$ and $p=10 \%$ corresponding to $6.7,8.8 \times 10^{20}$ and $1.1 \times 10^{21} \mathrm{~cm}^{-3}$.

We have observed an impurity band which was revealed by Angle-resolved photoemission spectroscopy[16], optical and transport measurements[17,18] and theoretical findings[4]. The results for $s_{h}$ are compared to the theoretical calculations of Ref. (3) given good agreement in the spontaneous magnetization region.

\section{ACKNOWLEDGEMENTS}

This work was partially supported by the Brazilian agencies FAPESB/PRONEX and CNPQ.

\section{REFERENCES}

1. A. H. Macdonald, P. Schiffer and N. Samarth, Nature Materials, 4, 195 (2005).

2. Dale Kitchen, Anthony Richardella, Jian-Ming Tang, Michael E. Flatté and Ali Yazdani Nature 442,446 (2006).

3. A. Ferreira da Silva, R. Kishore and I. C. da Cunha Lima, Phys. Rev. B 23, 4035 (1981).

4. M. Berciu and R. N. Bhatt, Phys. Rev. Lett. 87,107203 (2001);Phys. Rev. B 69, 045202 (2004).

5. A. Chattopadhyay, S. Das Sarma, and A. J. Millis, Phys. Rev. Lett. 87, 227202 (2001).

6. N.F. Mott, Can. J. Phys. 34, 1356 (1956).

7. K. F. Berggren, Philos. Mag. 27, 1027 (1973).

8. P. P. Edwards and M. J. Sienko, Phys. Rev. B 17, 2575 (1978).

9. P. Nubile and A. Ferreira da Silva, Solid State Electron 41, 121 (1997).

10. A. Ferreira da Silva, J. Appl. Phys. 79, 5249 (1994).

11. E. Abramof, A. Ferreira da Silva, B. E. Sernelius, J. P. de Souza and H. Boudinov, J. Mater. Res. 12, 641 (1997).

12. A. Ferreira da Silva, Phys. Scr., T14, 27 (1986)

13. T.Jungwirt, Jairo Sinova, A. H. MacDonald, B. L. Gallagher, V. Novák, K. W. Edmonds, A. W. Rushfort, R. P. Capion, C. T. Foxon, L. Eaves, E. Olejník, S. 
R. Eric Yang, J. Wundelich, C. Gould, L. W. Molenkamp, T. Dielt, and H. Ohno, Phys Rev. B 76, 125206 (2007).

14. A. Ferreira da Silva, I. Pepe, Bo E. Sernelius, C. Persson, R. Ahuja, J. P. de Souza, Yoko Suzuki, Y. Yang, J. Appl. Phys. 95, 2532 (2004)

15. Takeo Matsubara and Yutaka Toyozawa, Progr. Theor. Phys 26, 5 (1961).

16. J. Okabayashi, A. Kimura, O. Rader, T. Mizokawa, A. Fujimori, T. Hayashi and M. Tanaka, Physica E(Amsterdam) 10,192 (2001).

17. R. P. Campion, K. W. Edmonds, L. X. Zhao, K. Y. Wang., C. T. Foxon, B. L. Gallagher, and C. R. Staddon, J. Cryst. Growth 247, 42 (2003).

18. M. Kopecklý, J. Kub., E. Busetto, A. Lausi, M. Cukr, V. Novák, K. Olejnik, J. P. Wringht, and J. Fábry, J. Appl. Crystallog. 39, 735 (2006) 